\title{
Sequential and concomitant non-bismuth quadruple therapies are ineffective for $H$. pylori eradication in Palestine. A randomized trial ${ }^{*}$
}

\author{
Yasser Abu-Safieh", Hanin Yamin \\ Internal Medicine and GI Department, Specialized Arab Hospital, Affiliated with School of Medicine, An-Najah University, Nablus, \\ Palestine \\ Email: "yaserasaf@hotmail.com
}

Received 30 August 2012; revised 10 October 2012; accepted 20 October 2012

\begin{abstract}
Background: Increasing clarithromycin resistance has undermined the effectiveness of traditional clarithromycin-containing triple eradication therapy of Helicobacter pylori infections. Sequential and concomitant therapies show improved outcome with clarithromycin resistance. Aim: To evaluate the effectiveness of sequential and concomitant 4-drug nonbismuth therapies for eradication of Helicobacter pylori in a prospective, randomized, clinical trial conducted in Palestine. Patients and Methods: Patients who underwent upper endoscopy for a clinical indication and tested positive for rapid urease test were included. Subjects randomly allocated into two groups: One received a modified sequential therapy: esomeprazole $40 \mathrm{mg}$ OD and amoxicillin $1 \mathrm{~g}$ BID for 5 days then esomeprazole $40 \mathrm{mg}$ OD, clarithromycin $500 \mathrm{mg}$ BID and tinidazole $500 \mathrm{mg}$ BID for another 5 days. The other group received concomitant therapy in which the same $\mathbf{4}$ drugs and doses were all given daily for 10 days. Stool antigen was tested 4 weeks after completion of treatment. Results: Five hundred thirty three (533) patients were tested for $H$. pylori and 180 (34\%) were positive; 141 patients were included in the study and 112 patients completed. The overall per protocol eradication rate was $(74 \%$; $95 \% \mathrm{CI}=65.9 \%$ - 82.1\%). The eradication rates for sequential therapy was, $(\mathbf{7 0 . 9} \%$; $95 \% \mathrm{CI}=\mathbf{5 8 . 9} \%-\mathbf{8 2 . 9 \%})$ and for concomitant therapy $(77.2 \%$; $95 \% \mathrm{CI}=66.3 \%-88.1 \%)$. The results intention-to-treat were: sequential $61 \%$, concomitant $57 \%$. Conclusion: Neither sequential nor concomitant therapy achieved an acceptable $H$. pylori eradiation rate in Palestine.
\end{abstract}

Keywords: Helicobacter pylori; Eradication Therapies; Sequential Therapy; Concomitant Therapy

*No conflicts of interest exist.

\#Corresponding author.

\section{INTRODUCTION}

Helicobacter pylori infection causes peptic ulcers, gastric mucosa-associated lymphoid tissue lymphoma (MALT lymphoma), and gastric cancer [1]. Standard treatments for $H$. pylori infection endorsed by US and European authorities contain clarithromycin or metronidazole in conjunction with other antibiotics and acid inhibitors $[2,3]$. The prevalence of clarithromycin and metronidazole resistance has increased substantially in recent years along with a corresponding decrease in the eradication rate for $H$. pylori infections [4]. Eradication rates in most Western countries have generally declined to unacceptable levels such that therapy fails in approximately 1 in 5 patients [5]. A simple treatment regimen that would return eradication levels to those seen at the advent of $H$. pylori treatment is urgently needed [5]. Such a regimen should have high efficacy against clarithromycin-resistant and metronidazole-resistant strains of $H$. pylori because these strains are increasingly encountered in routine clinical practice.

One approach to the problem of clarithromycin resistance has been to administer the drugs sequentially $[6,7]$. The initial experiments with "sequential therapy" prescribed the dual therapy combination of amoxicillin and a PPI twice a day for 5 days followed by another 5 days of the PPI, plus clarithromycin and tinidazole/metronidazole. This approach has been compared with PPI amoxicillin plus clarithromycin triple therapy and repeatedly been shown to be superior [6-8]. The difference between the two approaches was related to improved results with clarithromycin resistant strains [6,7]. One potential problem with sequential therapy is that it is relatively complex requiring the patient to switch from a dual to a triple therapy at mid point $[6,9]$. It has been proposed that the same four drugs (a PPI, clarithromycin, metronidazole, and amoxicillin) can be given concomitantly as a non sequential four drug, three antibiotic non-bismuth containing quadruple therapy $[10,11]$, and 
clinical studies have shown that the efficacy of this regimen is equivalent to sequential therapy $[12,13]$. Palestine is a region where $H$. pylori infection and resistance to antimicrobial, among $H$. pylori strains, is thought to be high. These therapies have not formally been tested in Palestine. The aim of this study was to compare the efficacy of sequential and concomitant (non-bismuth) quadruple therapies for $H$. pylori eradication in a prospective, randomized, comparative clinical trial in Palestine.

\section{MATERIALS AND METHODS}

\subsection{Patients and Medications}

This study was a randomized trial performed at the GI clinic in Specialized Arab Hospital, Nablus, Palestine between April 2010 and January 2012. Patients presenting with dyspepsia or epigastric pain, and underwent upper endoscopy, with 2 antral biopsies. Those who tested positive for $H$. pylori by rapid urease testing (RUT) were included in this study. The exclusion criteria were: 1 ) patients younger than 18 years; 2) allergy to the antibiotics; 3 ) taking antibiotic or PPI within the 2 weeks before testing; and 4) active upper GI bleeding.

The study was approved by IRB, School of Medicine, An Najah University, and all subjects signed informed written consent after the study had been explained to them. Those with a positive RUT and meeting the entry criteria were randomly allocated into two groups: a group receiving sequential therapy consisting of esomeprazole $40 \mathrm{mg}$ od and amoxicillin $1 \mathrm{~g}$ bid for 5 days then esomeprazole $40 \mathrm{mg}$ OD, clarithromycin $500 \mathrm{mg}$, and tinidazole $500 \mathrm{mg}$ both given bid for another 5 days. The other group received concomitant therapy consisting of esomeprazole $40 \mathrm{mg}$ OD (once daily), amoxicillin $1 \mathrm{~g}$, tinidazole $500 \mathrm{mg}$, and clarithromycin $500 \mathrm{mg}$, all given bid, for 10 days. Eradication of $H$. pylori was assessed; by stool antigen testing was done by Specialized Arab Hospital Lab. (ACON Labs, Foresight ${ }^{\circledR}$ H. pylori Antigen EIA Test Kit) done four weeks after completion of treatment. Randomization was done by sealed opaque envelopes, with a given number for each patient, was done by our medical secretary.

\subsection{Statistical Analysis}

Per protocol analysis was used as the primary comparison of the eradication rates among the treatment regimens. Intention-to-treat included all subjects who received at least one dose of medication. SPSS version 15 was used in data analysis. Continuous variables were presented using mean and standard deviation and frequency tables were used to describe categorical variables. $\mathrm{X}^{2}(\mathrm{P}<0.05)$ was used. With confidence interval $(\mathrm{CI}=$ $95 \%)$.

\section{RESULTS}

The total number of unique patients who underwent upper endoscopy during the study period was 1122 and 533 were tested by RUT. The total number of positive RUTs was 180 (34\%); 39 patients were excluded for reasons listed in the methods. A total of 141 patients were included in the study and 112 patients (79\%) completed the study. As shown in Tables 1 and 2, the two groups did not differ significantly in age, sex, endoscopic findings, or dropout rates.

The overall PP eradication rate was 83/112 (74\%; 95\% $\mathrm{CI}=65.88 \%-82.12 \%)$. In per protocol analysis, the eradication rates were 39/55 (70.9\%; 95\% CI $=58.9 \%$ $82.9 \%)$ and $44 / 57$ (77.2\%; 95\% CI $=66.3 \%-88.1 \%)$ in those receiving sequential or concomitant therapies (p value $=0.45$ ). The ITT eradication rates were 39/64 (61\%), 44/77 (57\%) for sequential or concomitant therapies, respectively.

It was found that the overall eradication rate was $76.6 \%$ for female and $69.8 \%$ for males $(\mathrm{p}=0.325)$.

Patient compliance with the therapies was very good (the patients were seen 10 days after starting the regimen for 2 main reasons, 1-side effects of therapy, and 2compliance, we count how many tablets left, it was writ-

Table 1. Demographic characteristics of patients at entry at each treatment group.

\begin{tabular}{lcc}
\hline Patients characteristics (n) & Sequential & Concomitant \\
\hline $\begin{array}{l}\text { Number of patients enrolled } \\
\text { in the study (141) }\end{array}$ & 64 & 77 \\
$\begin{array}{l}\text { Number of patients } \\
\text { completed the study (112) }\end{array}$ & 55 & 57 \\
$\begin{array}{l}\text { Age (yr) mean } \pm \\
\text { standard deviation }\end{array}$ & $38.4 \pm 13$ & 41.412 \\
Sex (F/M) \% & $(50.9 / 49.1)$ & $(47.2 / 52.8)$ \\
Dropout, n (\%) & $9(14 \%)$ & $20(26 \%)$ \\
\hline
\end{tabular}

Table 2. Clinical characteristics of patients at entry at each treatment group.

\begin{tabular}{ccc}
\hline \multirow{2}{*}{ Endoscopic diagnosis } & Sequential & Concomitant \\
\cline { 2 - 3 } & Number (\%) & Number (\%) \\
\hline Gastritis & $32(58.2)$ & $24(42.9)$ \\
Duodenitis & $8(14.5)$ & $10(17.9)$ \\
Gastric ulcer & $4(7.3)$ & $4(7.1)$ \\
Duodenal ulcer & $16(29.1)$ & $11(25.6)$ \\
GERD & $11(20)$ & $12(21.4)$ \\
Gastric cancer & $0(0)$ & $1(1.8)$ \\
Candida esophagitis & $3(5.5)$ & $1(1.8)$ \\
\hline
\end{tabular}


Table 3. Antibiotic resistance in $H$. pylori isolates from selected countries in Middle East.

\begin{tabular}{ccccc}
\hline Country & Number tested & \multicolumn{3}{c}{ Antibiotics } \\
\hline Middle East & & Amoxicillin & Metronidazole & Clarithromycin \\
Bahrain & 83 & - & 57 & 33 \\
Egypt & 42 & 0 & 100 & 3 \\
Iran & 112 & - & 42 & - \\
Israel & 138 & 0.8 & $40-60$ & 10 \\
Lebanon & 54 & 0 & 30 & 2 \\
Saudi & 223 & 1.3 & 80 & 4 \\
UAE & 16 & - & 63 & - \\
\hline
\end{tabular}

ten on each prescription, the total number of tablets of each drug) and not different among the two groups. The complete follow up rate was $79 \%$. No serious side effects were reported by the patients.

\section{DISCUSSION}

The aim of this study was to compare the efficacy of sequential and concomitant therapies to determine if either was suitable for use in Palestine. Somewhat surprisingly, neither proved effective as the eradication rates achieved by the two protocols were unacceptably low. For triple therapy the eradication rate declined to unacceptable lower rates in most countries by the early 2000s [14]. The eradication rates of sequential and concomitant therapies obtained in this study were lower than most other countries $[8,13,15-18]$. The rationale for the different eradication success rates in different areas of the world can most likely be attributed to differences in the prevalence of resistance.

In regard to clarithromycin resistance in the Middle East; resistance in Bahrain was 33\% [19], in Egypt 3\% [19], in Israel 10\% [19], in Lebanon 2\% [19], in Saudi Arabia 4\% [19] and in Iran 23\% [20]. See Table 3.

Studies evaluating concomitant regimen from Europe, Asia, and North America from 19 studies (2070 patients) revealed a mean cure rate (intension-to-treat) of $88 \%$ [21]. However, a recent study from Latin America by Greenberg et al., had disappointing results with a 74\% eradication rate for a 5 day concomitant regimen [22]. Another negative study was in Turkey showed a 75\% eradication rate with a higher dose of metronidazole and 14 days duration of treatment [23]. And another study performed in Korea by Choi et al. achieved only a $63 \%$ cure rate in (38) patients [24].

In Palestine clarithromycin-containing therapies provide ineffective results for $H$. pylori eradication. We have an ethical question, is it ethical to use any of these therapies? Susceptibility testing is currently not available and trial and error are required to identify locally effective regimens.

\section{CONCLUSION}

H. pylori is still a major health problem in our region (34\% prevalence in this study) and neither sequential nor concomitant (non-bismuth quadruple therapies) proved useful here. Additional studies with different antibiotics will be needed to identify any regimen that provides an acceptable eradication rate.

\section{THE AUTHORS' CONTRIBUTION}

1) Dr. Abu-Safieh, all patient were seen and evaluated by me and endoscopy and RUT, biopsy was done by me and interpretation of the test, inclusion and exclusion of the cases, and follow up of symptoms after treatment,

2) Dr. Yamin, my resident, she collected and reviewed the literature put up the references and shared in writing the proposal and doing most of the statistical analysis. Follow up the stool Ag results.

Randomization was done by our medical secretary, she was given equal number of 2 prescriptions, A, B, (Sequential, and concomitant respectively), sealed opaque envelop. No one of the authors knew the treatment which was given when the patient is coming for follow up. We got to know it after stool Ag results.

The Institutional Research Board (IRB) of school of medicine in An-Najah University, approved the study before starting. (I am teaching GI and internal Medicine in the same university).

The procedure and the research were explained to the patients and a written consent signed.

\section{ACKNOWLEDGEMENTS}

We sincerely thank the lab staff of Specialized Arab Hospital for their efforts in stool antigen analysis. Besides, we would like to thank the medical secretaries for their invaluable help in documenting data and phoning the patients.

\section{REFERENCES}

[1] Suerbaum, S. and Michetti, P. (2002) Helicobacter pylori 
infection. The New England Journal of Medicine, 347, 1175-1186. doi:10.1056/NEJMra020542

[2] European Helicobacter Pylori Study Group (EHPSG) (2002) Current concepts in the management of Helicobacter pylori infection-The Maastricht 2-2000 consensus report. Alimentary Pharmacology \& Therapeutics, 16, 167-180. doi:10.1046/j.1365-2036.2002.01169.x

[3] Howden, C.W. and Hunt, R.H. (1998) Guidelines for the management of Helicobacter pylori infection. Ad Hoc committee on practice parameters of the American college of gastroenterology. American Journal of Gastroenterology, 93, 2330-2338. doi:10.1111/j.1572-0241.1998.00684.x

[4] Megraud, F. (2004) H. pylori antibiotic resistance: Prevalence, importance, and advances in testing. Gut, 53, 1374- 1384. doi:10.1136/gut.2003.022111

[5] Vakil, N. (2006) Helicobacter pylori treatment: A practical approach. American Journal of Gastroenterology, 101, 497-499. doi:10.1111/j.1572-0241.2006.00454.x

[6] Moayyedi, P. (2007) Sequential regimens for Helicobacter pylori eradication. Lancet, 370, 1010-1012. doi:10.1016/S0140-6736(07)61455-X

[7] Zullo, A., De, F.V., Hassan, C., Morini, S. and Vaira, D. (2007) The sequential therapy regimen for Helicobacter pylori eradication: A pooled-data analysis. Gut, 56, 13531357. doi:10.1136/gut.2007.125658

[8] Jafri, N.S., Hornung, C.A. and Howden, C.W. (2008) Meta-analysis: Sequential therapy appears superior to standard therapy for Helicobacter pylori infection in patients naive to treatment. Annals of Internal Medicine, 148, 923-931.

[9] Graham, D.Y., Lu, H. and Yamaoka, Y. (2008) Therapy for Helicobacter pylori infection can be improved: Sequential therapy and beyond. Drugs, 68, 725-736. doi:10.2165/00003495-200868060-00001

[10] Treiber, G., Ammon, S., Schneider, E., et al. (1998) Amoxicillin/metronidazole/omeprazole/clarithromycin: A new, short quadruple therapy for Helicobacter pylori eradication. Helicobacter, 3, 54-58. doi:10.1046/j.1523-5378.1998.08019.x

[11] Okada, M., Oki, K., Shirotani, T., et al. (1998) A new quadruple therapy for the eradication of Helicobacter pylori. Effect of pretreatment with omeprazole on the cure rate. Journal of Gastroenterology, 33, 640-645. doi:10.1007/s005350050150

[12] Wu, D.C., Hsu, P.I., Wu, J.Y., et al. (2010) Sequential and concomitant therapy with four drugs is equally effective for eradication of $H$. pylori infection. Clinical Gastroenterology and Hepatology, 8, 36-41. doi:10.1016/j.cgh.2009.09.030
[13] Yanai, A., Sakamoto, K., Akanuma, M., et al. (2012) Non Bismuth quadruple therapy for first line Helicobacter pylori eradication: A randomized study in Japan. World Journal of Gastrointestinal Pharmacology and Therapeutics, 3, 1-6.

[14] Graham, D.Y. and Fischbach, L. (2010) Helicobacter pylori treatment in the era of increasing antibiotic resistance. Gut, 59, 1143-1153. doi:10.1136/gut.2009.192757

[15] Okada, M., Nishimura, H., Kawashima, M., et al. (1999) A new quadruple therapy for Helicobacter pylori: Influence of resistant strains on treatment outcome. Alimentary Pharmacology \& Therapeutics, 13, 769-774. doi:10.1046/j.1365-2036.1999.00551.X

[16] Essa, A.S., Kramer, J.R., Graham, D.Y. and Treiber, G. (2009) Meta-analysis: Four-drug, three-antibiotic, nonbismuth-containing "concomitant therapy" versus triple therapy for Helicobacter pylori eradication. Helicobacter, 14, 109-118. doi:10.1111/j.1523-5378.2009.00671.x

[17] Sanchez-Delgade, J., Calvet, X., et al. (2008) Ten-day sequential treatment for Helicobacter pylori eradication in clinical practice. American Journal of Gastroenterology, 103, 2220-2223. doi:10.1111/j.1572-0241.2008.01924.x

[18] Gao, X.-Z., et al. (2010) Standard triple, bismuth pectin quadruple and sequential therapies for Helicobacter pylori eradication. World Journal of Gastroenterology, 16, 4357-4362. doi:10.3748/wjg.v16.i34.4357

[19] Gisbert, J.P. and Calvet, X. (2012) Update on non-bismuth quadruple (concomitant) therapy for eradication of Helicobacter pylori. Clinical and Experimental Gastroenterology, 5, 23-34. doi:10.2147/CEG.S25419

[20] Robert, W., Frenck, Jr. and Clemens, J. (2003) Helicobacter in the developing world. Microbes and Infection, 5, 705-713. doi:10.1016/S1286-4579(03)00112-6

[21] Tomatari, F., Mobarez, A., Amini, A., et al. (2010) Helicobacter pylori resistance to metronidazole and clarithromycin in dyspepsic patients in Iran. Iranian Red Crescent Medical Journal, 12, 409-412.

[22] Greenberg, E.R., Anderson, G.L., Morgan, D.R., et al. (2011) 14-day triple, 5-day concomitant, and 10-day sequential therapies for Helicobacter pylori infection in seven Latin American sites: A randomised trial. Lancet, 378, 507-514. doi:10.1016/S0140-6736(11)60825-8

[23] Toros, A.B., Ince, A.T., Kesici, B., et al. (2011) A new modified concomitant therapy for Helicobacter pylori eradication in Turkey. Helicobacter, 16, 225-228. doi:10.1111/j.1523-5378.2011.00823.x

[24] Choi, C., Lee, D., Chon, I., et al. (2011) Concomitant therapy was more effective than ppi-base triple therapy in Korea: A preliminary report. Helicobacter, 16, 136. 